\title{
Blocking Parameterizations for Improving the Computational Tractability of Affine Disturbance Feedback MPC Problems
}

\author{
Frauke Oldewurtel, Ravi Gondhalekar, Colin N. Jones, Manfred Morari
}

\begin{abstract}
Many robust model predictive control (MPC) schemes suffer from high computational complexity. Especially robust MPC schemes, which explicitly account for the effects of disturbances, can result in computationally intractable problems. So-called move-blocking is an effective method of reducing the computational complexity of MPC problems. Unfortunately move-blocking precludes the use of terminal constraints as a means of enforcing strong feasibility of MPC problems. Thus move-blocking MPC has traditionally been employed without rigorous guarantees of constraint satisfaction. A method for enforcing strong feasibility of nominal move-blocking MPC problems was recently developed. The contribution of this paper is to generalize this method and employ it for the purpose of enforcing strong feasibility of move-blocking affine disturbance feedback robust MPC problems. Furthermore the effectiveness of different disturbance-feedback blocking strategies is investigated by means of a numerical example.
\end{abstract}

Keywords: Robust control; Model predictive control; Affine disturbance feedback; Controlled invariant feasibility

\section{INTRODUCTION}

This paper deals with solving a robust MPC problem for the class of constrained discrete-time linear systems subject to additive, bounded disturbances. The optimal solution is given by dynamic programming, this is however not tractable except for very small problems or under special circumstances. An effective approach of approximating dynamic programming for this class of systems is to apply affine disturbance feedback (ADF), which is often close to optimal but computationally demanding for long prediction horizons $[10,8]$. The motivation for this paper is to reduce the number of degrees of freedom of the disturbance feedback parameterization for the purpose of reducing the computational complexity of the resulting MPC problem, and furthermore to investigate how to reduce the degrees of freedom in such a way as to maintain high control performance.

\section{A. Affine Disturbance Feedback Parameterization}

In so-called open-loop prediction (OLP) MPC the control action that is predicted to be taken in the future is only a function of the current state, which is computationally very attractive, but often results in very conservative control behavior. An alternative approach, proposed in [1] and [9] is to apply closed-loop prediction MPC by employing an affine disturbance feedback parameterization of the control inputs,

Frauke Oldewurtel, Colin N. Jones and Manfred Morari are with the Automatic Control Laboratory, Department of Electrical Engineering, Swiss Federal Institute of Technology in Zurich (ETHZ), Switzerland.

\{oldewurtel, cjones, morari\}@control.ee.ethz.ch

Ravi Gondhalekar is with the Frontier Research Base for Global Young Researchers and Dept. of Mechanical Engineering, Osaka University, Japan. ravi.gondhalekar@wakate.frc.eng.osaka-u.ac.jp which was shown to be equivalent to affine state feedback and is often close to optimal [8]. In contrast to the affine state feedback, the affine disturbance feedback parameterization leads to a convex set of feasible decision variables. The computational complexity is, however, increased compared to open-loop prediction, because of a significant increase in the number of decision variables. Our aim is to reduce the computational complexity of the affine disturbance feedback while maintaining good control performance.

\section{B. Blocking for Reducing Computational Complexity}

In order to reduce the computational complexity, it is common practice in nominal MPC to reduce the number of decision variables by fixing the input or its derivatives to be constant over several prediction steps. Although this alleviates the computational burden, this renders terminal constraints incapable of enforcing strong feasibility and stability and often leads to sub-optimality. In [4] the authors propose to employ a time-varying blocking matrix such that stability can be guaranteed. In $[7,6]$ strong feasibility is guaranteed with a time-invariant blocking matrix by constraining the state at the first prediction step to a controlled invariant feasible set. In contrast to [4] this method is guaranteed to be least-restrictive. In order to simplify the computation of the controlled invariant feasible set, the approach from [6] is used, where a controlled invariant feasible set is computed by taking a controlled invariant set and relaxing the state constraints of the prediction problem so as to render the controlled invariant set a controlled invariant feasible set.

\section{Main Idea and Outline}

In this paper we impose a structure on the disturbance feedback matrix in such a way that the resulting loss in performance is small. Furthermore, we extend the method in [6] to the robust case to guarantee strong feasibility. In Section II the problem setting is given, the affine disturbance feedback parameterization is described in detail, and the MPC problem with an expectation based cost is formulated. Section III introduces the concept of blocking for the affine disturbance feedback matrix and explains how strong feasibility can be guaranteed. The proposed method is demonstrated in an illustrative example in Section IV and a conclusion is given in Section V.

\section{Notation}

The real number set is denoted by $\mathbb{R}$, the set of nonnegative integers by $\mathbb{N}\left(\mathbb{N}_{+}:=\mathbb{N} \backslash\{0\}\right)$, the set of consecutive non-negative integers $\{j, \ldots, k\}$ by $\mathbb{N}_{j}^{k}$. Denote by $I_{n} \in$ 
$\{0,1\}^{n \times n}$ the identity matrix, by $0_{\{n, m\}} \in\{0\}^{n \times m}$ the zero matrix and by 0 without subscript the zero matrix with dimension deemed obvious by context. For matrices $A$ and $B$ of equal dimension inequalities $A\{<, \leq,>, \geq\} B$ hold component-wise. The expectation of a stochastic variable $w$ is denoted by $\mathrm{E}[w]$. For a set $\mathbb{X}$ the set of non-empty subsets, i.e. the power-set less $\emptyset$, is denoted by $2^{\mathbb{X}}$. The future value of a variable $\psi$ at step $i+k$, as predicted from step $i$ is denoted by $\psi_{(i, k)}$. For compact notation $\psi_{(i, 0)}=\psi_{i}$.

\section{Affine Disturbance FeEdback MPC}

\section{A. Problem Setting}

Consider the discrete-time linear time-invariant system

$$
x_{i+1}=A x_{i}+B u_{i}+E w_{i}
$$

with step index $i \in \mathbb{N}$, state $x \in \mathbb{R}^{n}, n \in \mathbb{N}_{+}$, control input $u \in \mathbb{R}^{m}, m \in \mathbb{N}_{+}$and disturbance $w \in \mathbb{R}^{p}, p \in \mathbb{N}_{+}$.

Assumption 1: The pair $(A, B)$ is stabilizable.

State $x$ and control input $u$ must satisfy constraints

$$
x_{i} \in \mathcal{X} \in 2^{\mathbb{R}^{n}} \quad \wedge \quad u_{i} \in \mathcal{U} \in 2^{\mathbb{R}^{m}} \quad \forall i \in \mathbb{N} .
$$

Assumption 2: Disturbances satisfy $w_{i} \in \mathcal{W} \in 2^{\mathbb{R}^{p}} \forall i \in \mathbb{N}$.

Assumption 3: Constraint sets $\mathcal{X}, \mathcal{U}$ and $\mathcal{W}$ have nonempty interiors and are polytopic (polyhedral, convex and bounded), time-invariant, and known.

For some initial state $x_{0} \in \mathbb{R}^{n}$ the control objective is to minimize the quadratic cost function $V: \mathbb{R}^{n} \rightarrow \mathbb{R}$,

$$
V\left(x_{0}\right):=\sum_{i=0}^{\hat{i}}\left[x_{i}^{\mathrm{T}} Q x_{i}+u_{i}^{\mathrm{T}} R u_{i}\right]
$$

with $Q \in \mathbb{R}^{n \times n}, Q=Q^{\mathrm{T}} \succeq 0, R \in \mathbb{R}^{m \times m}, R=R^{\mathrm{T}} \succ 0$, where $\hat{i} \in \mathbb{N}_{+}$is some desired number of steps, i.e. the window of interest.

\section{B. Affine Disturbance Feedback Input Parameterization}

Consider a prediction horizon $N \in \mathbb{N}_{+}$and define

$$
\begin{aligned}
\mathbf{x}_{i} & :=\left[x_{(i, 0)}^{\mathrm{T}}, \ldots, x_{(i, N)}^{\mathrm{T}}\right]^{\mathrm{T}} \in \mathbb{R}^{(N+1) n} \\
\mathbf{u}_{i} & :=\left[u_{(i, 0)}^{\mathrm{T}}, \ldots, u_{(i, N-1)}^{\mathrm{T}}\right]^{\mathrm{T}} \in \mathbb{R}^{N m} \\
\mathbf{w}_{i} & :=\left[w_{(i, 0)}^{\mathrm{T}}, \ldots, w_{(i, N-1)}^{\mathrm{T}}\right]^{\mathrm{T}} \in \mathbb{R}^{N p}
\end{aligned}
$$

and prediction dynamics matrices $\mathbf{A}, \mathbf{B}$ and $\mathbf{E}$ such that

$$
\mathbf{x}_{i}=\mathbf{A} x_{i}+\mathbf{B} \mathbf{u}_{i}+\mathbf{E} \mathbf{w}_{i} .
$$

Assumption 4: The following statements hold $\forall i \in \mathbb{N}$ :

$$
\begin{aligned}
\mathbf{w}_{i} & \in \mathscr{W}:=\mathcal{W}^{N} \\
\mathrm{E}\left[\mathbf{w}_{i}\right] & :=\overline{\mathbf{w}}_{i} \in \mathbb{R}^{N p} \\
\mathrm{E}\left[\left(\mathbf{w}_{i}-\overline{\mathbf{w}}_{i}\right)\left(\mathbf{w}_{i}-\overline{\mathbf{w}}_{i}\right)^{\mathrm{T}}\right] & :=\Sigma_{i}=\Sigma_{i}^{\mathrm{T}} \in \mathbb{R}^{N p \times N p} .
\end{aligned}
$$

Note that the predicted disturbance trajectories $\mathbf{w}_{i}$ as well as their stochastic properties $\overline{\mathbf{w}}_{i}$ and $\Sigma_{i}$ may change from one step $i$ to the next $i+1$. The stochastic properties are assumed known, the disturbances themselves not. The constraint set
$\mathscr{W}$ is time-invariant and known by Assumption 3. This is critical for the proposed approach.

In affine disturbance feedback MPC $[8,11]$ the control input trajectory $\mathbf{u}_{i}$ is parameterized such that statements

$$
\begin{aligned}
u_{(i, k)} & =h_{(i, k)}+\sum_{j=0}^{k-1} M_{(i,[k, j])} w_{(i, j)} \\
h_{(i, k)} & \in \mathbb{R}^{m} \\
M_{(i,[k, j])} & \in \mathbb{R}^{m \times p} \quad \forall j \in \mathbb{N}_{0}^{k}
\end{aligned}
$$

hold $\forall(i, k) \in \mathbb{N} \times \mathbb{N}_{0}^{N-1}$. In matrix form this leads to

$$
\begin{aligned}
\mathbf{u}_{i} & =\mathbf{M}_{i} \mathbf{w}_{i}+\mathbf{h}_{i} \\
\mathbf{M}_{i} & :=\left[\begin{array}{cccc}
0 & \ldots & \ldots & 0 \\
M_{(i,[1,0])} & 0 & \cdots & 0 \\
\vdots & \ddots & \ddots & \vdots \\
M_{(i,[N-1,0])} & \cdots & M_{(i,[N-1, N-2])} & 0
\end{array}\right] \\
& \in \mathbb{R}^{N m \times N p} \\
\mathbf{h}_{i} & :=\left[h_{(i, 0)}^{\mathrm{T}}, \ldots, h_{(i, N-1)}^{\mathrm{T}}\right]^{\mathrm{T}} \in \mathbb{R}^{N m} .
\end{aligned}
$$

Consider the quadratic cost function

$$
J\left(x_{i}, \mathbf{M}_{i}, \mathbf{h}_{i}, \mathbf{w}_{i}\right):=\mathbf{x}_{i}^{\mathrm{T}} \mathbf{Q} \mathbf{x}_{i}+\mathbf{u}_{i}^{\mathrm{T}} \mathbf{R} \mathbf{u}_{i}
$$

where $\mathbf{Q} \in \mathbb{R}^{(N+1) n \times(N+1) n}, \mathbf{Q}=\mathbf{Q}^{\mathrm{T}} \succeq 0$ and $\mathbf{R} \in$ $\mathbb{R}^{N m \times N m}, \mathbf{R}=\mathbf{R}^{\mathrm{T}} \succ 0$ contain all stage and terminal costs, and further consider prediction constraints

$$
\mathbf{x}_{i} \in \mathcal{X}^{N+1} \wedge \mathbf{u}_{i} \in \mathcal{U}^{N} \quad \forall i \in \mathbb{N} .
$$

For state $x_{i}$ the set of admissible affine disturbance feedback policies $\left(\mathbf{M}_{i}, \mathbf{h}_{i}\right)$ is then given by the set [8]

$\Pi\left(x_{i}\right):=\left\{\begin{array}{l|l}(\mathbf{M}, \mathbf{h}) \mid \begin{array}{l}\mathbf{M} \text { satisfies Eq. (4), } \\ \mathbf{A} x_{i}+\mathbf{B h}+(\mathbf{B M}+\mathbf{E}) \mathbf{w} \in \mathcal{X}^{N+1} \\ \wedge \mathbf{M} \mathbf{w}+\mathbf{h} \in \mathcal{U}^{N} \quad \forall \mathbf{w} \in \mathscr{W}\end{array}\end{array}\right\}$.

Define the set $\mathbb{X}_{0}$ of states $x$ for which an admissible affine disturbance feedback policy $(\mathbf{M}, \mathbf{h})$ exists by

$$
\mathbb{X}_{0}:=\left\{x \in \mathbb{R}^{n} \mid \Pi(x) \neq \emptyset\right\}
$$

The objective in this paper is to apply a blocking parameterization of the decision variables and on guaranteeing strong feasibility of the MPC problem.

\section{Expectation Based Disturbance Feedback MPC Problem}

The optimal control input $u_{i}$ is determined by solving MPC Problem 1.

Problem 1: Determine:

$$
\left(\mathbf{M}^{*}\left(x_{i}\right), \mathbf{h}^{*}\left(x_{i}\right)\right):=\arg \min _{(\mathbf{M}, \mathbf{h}) \in \Pi\left(x_{i}\right)} \mathrm{E}\left[J\left(x_{i}, \mathbf{M}, \mathbf{h}, \mathbf{w}\right)\right] .
$$

Remark 1: The expectation $\mathrm{E}[J]$ of the quadratic cost is employed as the optimization objective. Thus the stochastic properties $\left(\overline{\mathbf{w}}_{i}, \Sigma_{i}\right)$ of disturbance trajectory $\mathbf{w}_{i}$ are taken into account, even though they do not appear explicitly in MPC Problem 1. More usually the nominal cost is used, which is equivalent to the expectation with $\overline{\mathbf{w}}_{i}=0 \wedge \Sigma_{i}=0 \forall i \in \mathbb{N}$.

The optimal control input $u_{i}^{*}\left(x_{i}\right)$ is given by

$$
u_{i}^{*}\left(x_{i}\right)=h_{(i, 0)}^{*}\left(x_{i}\right)
$$


and the closed-loop state trajectory evolves according to

$$
x_{i+1}=A x_{i}+B u_{i}^{*}\left(x_{i}\right)+E w_{i} \quad \forall i \in \mathbb{N} .
$$

Definition 1: An MPC problem is strongly feasible if and only if from every feasible state the closed-loop state trajectory due to any sequence of feasible solutions, and due to any admissible disturbances, remains within the feasible set.

For example, MPC Problem 1 is strongly feasible if and only if the following condition holds:

$$
\begin{aligned}
& x_{i+1}=A x_{i}+B h_{(i, 0)}\left(x_{i}\right)+E w_{i} \in \mathbb{X}_{0} \\
& \forall w_{i} \in \mathcal{W} \quad \forall\left(\mathbf{M}_{i}, \mathbf{h}_{i}\right) \in \Pi\left(x_{i}\right) \quad \forall i \in \mathbb{N} \quad \forall x_{0} \in \mathbb{X}_{0} .
\end{aligned}
$$

Definition 2: A least-restrictive strongly feasible MPC problem is one such that there exists no strongly feasible MPC problem with a larger feasible set, for the same system and constraints.

MPC Problem 1 can be reformulated into a standard QP problem, where the optimization variables are the vector $\mathbf{h}_{i}$ of offsets and a vector $\overline{\mathbf{M}}_{i} \in \mathbb{R}^{\frac{N(N-1) m p}{2}}$, which contains all decision variables of disturbance feedback matrix $\mathbf{M}_{i}$.

Remark 2: In affine disturbance feedback MPC at each step $i$ the feedback policies $(\mathbf{M}, \mathbf{h})$ are optimized, rather than predicted control actions $\mathbf{u}$ as in open-loop prediction MPC. Affine disturbance feedback MPC is generally capable of high performance. However, the number of decision variables of the MPC problem is of order $N^{2}$, due to disturbance feedback matrix $M$. The number of decision variables for open-loop prediction MPC is only of order $N$.

Remark 3: An open-loop prediction MPC problem can easily be derived from MPC Problem 1 by forcing $\mathbf{M}=$ 0 in both the cost function $J$ and the constraints $\Pi$. A consequence of this is that the set of feasible solutions of the open-loop prediction MPC problem is a subset (usually a strict subset) of the set of feasible solutions of the affine disturbance feedback MPC problem. Thus closedloop prediction MPC is expected to outperform open-loop prediction MPC. However, no statements about closed-loop performance can be made until after controller verification.

\section{Complexity Reduction Via Blocking}

\section{A. Blocking Parameterization}

To reduce the computational complexity of MPC Problem 1 the number of decision variables is reduced by employing a method called blocking. Traditionally a method called moveblocking is used in controller design, whereby the number of control moves is reduced by forcing the predicted control input trajectory to be constant over sets of multiple prediction steps [4]. In this paper the term blocking is used to mean that a vector of decision variables is parameterized by a lowerdimensional vector of decision variables in combination with a so-called blocking matrix, which forces some structure upon the original vector of decision variables.
The vector $\overline{\mathbf{M}}_{i}$ of decision variables of $\mathbf{M}_{i}$ is parameterized by blocking matrix $\Lambda$ and a vector $\tilde{\mathbf{M}}_{i}$ according to:

$$
\begin{aligned}
\overline{\mathbf{M}}_{i} & =\Lambda \tilde{\mathbf{M}}_{i} & & \\
\tilde{\mathbf{M}}_{i} & \in \mathbb{R}^{\nu} & & \Lambda \in\{0,1\} \frac{N(N-1) m p}{2} \times \nu \\
\nu & \in \mathbb{N} & & \nu \leq \frac{N(N-1) m p}{2} .
\end{aligned}
$$

Blocking matrix $\Lambda$ and dimension $\nu$ are design parameters and can be manipulated to affect the complexity of the blocked MPC problem. In this work only disturbance feedback matrix $M$ is parameterized, as opposed to offset vector $\mathbf{h}$ also. One reason for this is that $\mathbf{M}$ has the most decision variables, in general. Furthermore, by parameterizing $\mathbf{M}$ only the set of feasible solutions of the MPC problem is still guaranteed to be a superset (usually strict superset) of the set of feasible solutions of the open-loop prediction MPC problem, (see Remark 3).

Remark 4: A diagonal structure, i.e. letting $\mathbf{M}_{i}$ have the same sub-matrix blocks $\mathbf{M}_{(i,[k, j])}$ along the diagonals, such that $\mathbf{M}_{(i,[k+1, j+1])}=\mathbf{M}_{(i,[k, j])}$ implies that the disturbance feedback policy is time-invariant w.r.t. prediction time $k$ and appears to be effective in numerical trials.

\section{B. Prediction Constraint Relaxation}

The purpose of this section is to apply the method proposed in [6] for designing nominal, least-restrictive, strongly feasible blocking MPC problems to the robust, affine disturbance feedback setting. In non-blocking MPC problems, affine disturbance feedback or non, strong feasibility is usually enforced by constraining the state $x_{(i, N)}$ of the final prediction step to a robust invariant set $[3,8,10]$. This assumes that the shifted solution from the previous step is an admissible solution for the current step, up to but not including the final control move, and that subsequently the existence of the final control move is guaranteed by the robust invariant terminal constraints. When employing blocking this assumption no longer holds, firstly because due to the structure imposed on disturbance feedback matrix $\mathbf{M}$ the shifted solution from the previous step may not be an admissible solution at the current step. Secondly, even if it were, the final control move may not be free to be choosen, as it may be dependent on the previous control moves, depending on the particular blocking matrix $\Lambda$ selected.

The outline of the proposed procedure is as follows. To enforce strong feasibility of the MPC problem its constraints must directly enforce that the state at the next step remains within the feasible set, recursively. Thus the state $x_{(i, 1)}$ of the first prediction step is explicitly constrained to a robust controlled invariant set. However, feasibility of the blocked MPC problem is not guaranteed for each element of a robust controlled invariant set, because due to the blocking scheme the predicted state constraints may no longer be satisfiable. Therefore the prediction state constraints of steps beyond the first prediction step are relaxed, i.e. expanded, in a minimal way so as to admit a feasible solution to the MPC problem for each element of the robust controlled invariant set. Despite the relaxed prediction state constraints 
the constraints on the first prediction step enforce constraint satisfaction of the closed-loop state trajectory.

Definition 3: A non-empty set $\mathbb{C} \in 2^{\mathcal{X}}$ is a robust controlled invariant set for system (1) subject to constraints (2) if it satisfies the following condition [3]:

$\forall x \in \mathbb{C} \quad \exists u \in \mathcal{U}$ s.t. $A x+B u+E w \in \mathbb{C} \quad \forall w \in \mathcal{W}$.

The maximum robust controlled invariant (MRCI) set $\mathbb{C}^{*} \in$ $2^{\mathcal{X}}$ is the largest element in $2^{\mathcal{X}}$ with this property $[2,5]$.

It is implied throughout this paper that disturbance constraint set $\mathcal{W}$ is small enough such that $\mathbb{C}^{*} \neq \emptyset$. If this does not hold then any attempt at controller design is futile. Thus assume $\mathbb{C}^{*} \neq \emptyset$. Then, $\mathbb{C}^{*}$ is convex and bounded under Assumption 3. Thus if $\mathbb{C}^{*}$ is not a polytope then it can be under-approximated arbitrarily closely by a polytope. To facilitate a computationally viable controller design procedure make Assumption 5.

Assumption 5: MRCI set $\mathbb{C}^{*}$ has been determined and is given by the polytope

$$
\mathbb{C}^{*}:=\left\{x \in \mathbb{R}^{n} \mid Y x \leq y\right\},
$$

the number of vertices of $\mathbb{C}^{*}$ is $\kappa \in \mathbb{N}_{+}$, and vertices $\mathscr{V}_{j} \in$ $\mathbb{R}^{n} \forall j \in \mathbb{N}_{1}^{\kappa}$ have been computed.

Remark 5: The set $\mathbb{C}^{*}$ is employed to design strongly feasible MPC problems which contain blocking parameterizations. In fact the employed set need not be MRCI set $\mathbb{C}^{*}$, any robust controlled invariant set does suffice. However, use of $\mathbb{C}^{*}$ results in a least-restrictive, strongly feasible MPC problem. If the particular control problem at hand permits a restrictive controller then a non-maximum robust controlled invariant set can replace $\mathbb{C}^{*}$. In such cases subsequent results on least-restrictiveness do not apply. Subsequent results on strong feasibility do apply.

Let $\mathcal{X}:=\left\{x \in \mathbb{R}^{n} \mid C x \leq c\right\}, C \in \mathbb{R}^{t \times n}, c \in \mathbb{R}^{t}$. Prediction state constraints (5) are stated explicitly as

$$
C x_{(i, k)} \leq c \quad \forall k \in \mathbb{N}_{0}^{N} \quad \forall i \in \mathbb{N} .
$$

The aim is to compute the minimal prediction constraint relaxations $\zeta_{k} \in \mathbb{R}^{t} \wedge \zeta_{k} \geq 0 \forall k \in \mathbb{N}_{2}^{N}$ such that when the prediction state constraints enforced in the MPC problem are

$$
\begin{aligned}
& Y x_{(i, 1)} \leq y \\
& C x_{(i, k)} \leq c+\zeta_{k} \quad \forall k \in \mathbb{N}_{2}^{N},
\end{aligned}
$$

then the resulting MPC problem is feasible for every element $x$ of MRCI set $\mathbb{C}^{*}$. Robust controlled invariant constraint (7) directly enforces recursive constraints satisfaction of the actual closed-loop state trajectory. State constraints for prediction steps $k \in \mathbb{N}_{2}^{N}$ can be relaxed arbitrarily. To maintain good cost performance it is desirable to relax the prediction state constraints in a minimal way. Here, minimality is with respect to the 2-norm. Let

$$
\begin{aligned}
\zeta & :=\left[\zeta_{2}^{\mathrm{T}}, \ldots, \zeta_{N}^{\mathrm{T}}\right]^{\mathrm{T}} \in \mathbb{R}^{(N-1) t} \\
\mathcal{X}_{k} & :=\left\{x \in \mathbb{R}^{n} \mid C x \leq c+\zeta_{k}\right\} \quad \forall k \in \mathbb{N}_{2}^{N} \\
\mathscr{X} & :=\mathcal{X} \times \mathbb{C}^{*} \times \mathcal{X}_{2} \times \cdots \times \mathcal{X}_{N} \in 2^{\mathbb{R}^{(N+1) n}}
\end{aligned}
$$

where $\mathcal{X}_{k}$ denotes the relaxed prediction state constraint set for prediction step $k$, and $\mathscr{X}$ is the resulting constraint set for the entire predicted state trajectory $\mathbf{x}$. The minimal relaxation $\zeta^{*}$ is given by the solution of QP Problem 2.

Problem 2: Determine:

$$
\zeta^{*}:=\arg \min _{\zeta \in \mathbb{R}^{(N-1) t}} \zeta^{\mathrm{T}} \zeta
$$

subject to

$$
\begin{aligned}
\forall j \in \mathbb{N}_{1}^{\kappa} \quad \exists\left(\tilde{\mathbf{M}}_{j}, \mathbf{h}_{j}\right) \quad \text { s.t. } & \\
Y\left(A \mathscr{V}_{j}+B h_{(j, 0)}+E w_{(j, 0)}\right) \leq y & \wedge \\
\mathbf{A} \mathscr{V}_{j}+\mathbf{B h}_{j}+\left(\mathbf{B M}_{j}+\mathbf{E}\right) \mathbf{w} \in \mathscr{X} & \wedge \\
\mathbf{M}_{j} \mathbf{w}+\mathbf{h}_{j} \in \mathcal{U}^{N} & \forall \mathbf{w}_{j} \in \mathscr{W}
\end{aligned}
$$

where $\mathbf{M}_{j}$ results from $\tilde{\mathbf{M}}_{j}$ and blocking strategy (6).

The constraints of Problem 2 enforce that for each vertex $\mathscr{V}_{j}$ of MRCI set $\mathbb{C}^{*}$ there exists an admissible blocked feedback policy $\left(\tilde{\mathbf{M}}_{j}, \mathbf{h}_{j}\right)$.

Problem 2 can be reformulated as a QP problem (details omitted). Note that finding $\zeta^{*}$ requires simultaneous optimization over each vertex $\mathscr{V}_{j}$ of $\mathbb{C}^{*}$. Thus Problem 2 is very large for a large number of vertices. An over-approximation can be determined at much lower computational cost by finding a minimal relaxation $\zeta$ for each vertex $\mathscr{V}_{j}$ in turn, then maximizing over those solutions (details omitted). Note that under-approximations of $\zeta^{*}$ are not suitable. Also note that all further results hold when employing over-approximations.

Theorem 1: Problem 2 admits a feasible solution.

Proof: Constraint (9) implies robust controlled invariance. As the pair $(Y, y)$ describes MRCI set $\mathbb{C}^{*}$ the existence of a suitable $h_{(i, 0)}$ is guaranteed trivially. Next, because disturbance trajectory constraint set $\mathscr{W}$ is bounded the predicted state trajectory $\mathbf{x}_{j}$ from any vertex $\mathscr{V}_{j}$, for any disturbance sequence $\mathbf{w}_{j} \in \mathscr{W}$ and for any feedback policy $(\mathbf{M}, \mathbf{h})$, is bounded. Thus there exists a $\zeta$ satisfying $\zeta^{\mathrm{T}} \zeta<\infty$ such that $\mathbf{x}_{j} \in \mathscr{X} \forall j \in \mathbb{N}_{1}^{\kappa}$.

Let $\mathscr{X}^{*}$ be the minimally relaxed constraint set for predicted state trajectory $\mathbf{x}$, analogously as in Eq. (8). Incorporating prediction constraint relaxations the set $\tilde{\Pi}$ of admissible blocked disturbance feedback policies is given by

$\tilde{\Pi}\left(x_{i}\right):=\left\{(\tilde{\mathbf{M}}, \mathbf{h}) \mid \begin{array}{ll}\mathbf{A} x_{i}+\mathbf{B h}+(\mathbf{B M}+\mathbf{E}) \mathbf{w} \in \mathscr{X}^{*} \\ \wedge & Y\left(A x_{i}+B h_{(i, 0)}+E w_{(i, 0)}\right) \leq y \\ \wedge & \mathbf{M} \mathbf{w}+\mathbf{h} \in \mathcal{U}^{N} \quad \forall \mathbf{w} \in \mathscr{W}\end{array}\right\}$

where $\mathbf{M}$ results from $\tilde{\mathbf{M}}$ and blocking strategy (6).

The set $\tilde{\mathbb{X}}_{0}$ for which an admissible blocked affine disturbance feedback policy $(\tilde{\mathbf{M}}, \mathbf{h})$ exists is given by

$$
\tilde{\mathbb{X}}_{0}:=\left\{x \in \mathbb{R}^{n} \mid \tilde{\Pi}(x) \neq \emptyset\right\} .
$$

The MPC problem with constraint relaxations is given by MPC Problem 3, where the only difference is the optimization space. The prediction cost function remains unchanged.

Problem 3: Determine:

$$
\left(\tilde{\mathbf{M}}^{*}\left(x_{i}\right), \mathbf{h}^{*}\left(x_{i}\right)\right):=\arg \min _{(\tilde{\mathbf{M}}, \mathbf{h}) \in \tilde{\Pi}\left(x_{i}\right)} \mathrm{E}\left[J\left(x_{i}, \mathbf{M}, \mathbf{h}, \mathbf{w}\right)\right]
$$


where once again $\mathbf{M}$ results from $\tilde{\mathbf{M}}$.

Theorem 2: The following statements hold:

(1) $\tilde{\mathbb{X}}_{0}=\mathbb{C}^{*}$

(2) MPC Problem 3 is strongly feasible.

(3) MPC Problem 3 is a least-restrictive MPC problem for system (1) subject to constraints (2).

Proof: (1) A formal proof is omitted, but this follows from the convexity of $\mathbb{C}^{*}, \mathcal{U}, \mathcal{X}, \mathcal{W}$, and from linearity of system (1). We first show that $\tilde{\mathbb{X}}_{0} \supseteq \mathbb{C}^{*}$. Any $x \in \mathbb{C}^{*}$ can be written as a convex sum of the vertices $\mathscr{V}_{j}$ of $\mathbb{C}^{*}$. Problem 2 determines the relaxation $\zeta^{*}$ such that a feedback policy $\left(\tilde{\mathbf{M}}_{j}, \mathbf{h}_{j}\right)$ which satisfies the prediction constraints exists $\forall j \in \mathbb{N}_{1}^{\kappa}$. For all states $x \in \mathbb{C}^{*}$ there then exists a convex sum of these feedback policies which is admissible. We next show that $\tilde{\mathbb{X}}_{0} \subseteq \mathbb{C}^{*}$. Constraint (9) explicitly forces $\tilde{\mathbb{X}}_{0}$ to be a robust controlled invariant set. Thus clearly it must be a subset of maximum controlled invariant (MCI) set $\mathbb{C}^{*}$.

(2) For any initial state $x_{0} \in \mathbb{C}^{*}$ the constraint set $\mathscr{X}^{*}$ directly enforces that $x_{1}=x_{(0,1)} \in \mathbb{C}^{*}$ for any admissible feedback policy $(\tilde{\mathbf{M}}, \mathbf{h}) \in \tilde{\Pi}\left(x_{0}\right)$ and despite any admissible disturbance $\mathbf{w}_{0} \in \mathscr{W}$. By induction this holds recursively.

(3) By the definition of MRCI set $\mathbb{C}^{*}$ a strongly feasible MPC problem with a feasible set larger than $\mathbb{C}^{*}$ cannot exist. Thus, due to Theorem 2 (1) above, MPC Problem 3 is leastrestrictive.

Remark 6: The proposed method is especially beneficial for long prediction horizons $N$, since the computational complexity of affine disturbance feedback grows with the order of $N^{2}$ whereas the computation of the robust controlled invariant set does not depend on $N$ and moreover has to be done just once.

\section{Numerical EXAMPle - The Integrated CANine}

A man of mass $m_{1}=70 \mathrm{Kg}$ is walking his dog of mass $m_{2}=10 \mathrm{Kg}$ along a $3 \mathrm{~m}$ wide sidewalk. To the right of the sidewalk is a road, to the left a muddy field. In the field, $6 \mathrm{~m}$ from the edge of the sidewalk, is a cat. The dog notices the cat and attempts to attack it. Luckily for the cat the dog's leash is shorter than the distance from the edge of the field to the cat. The dog is pulling on the leash in order to get to the cat. However, the man does not want to get muddy shoes, therefore pulls the dog back in order to avoid being pulled into the field. He cannot pull back too far as he must avoid entering the road to the rear.

A model of this problem is depicted in Figure 1. The man's position is denoted by $x$. A position $x>x_{\max }=9 \mathrm{~m}$ implies the man is on the road, whereas $x<x_{\min }=6 \mathrm{~m}$ implies he

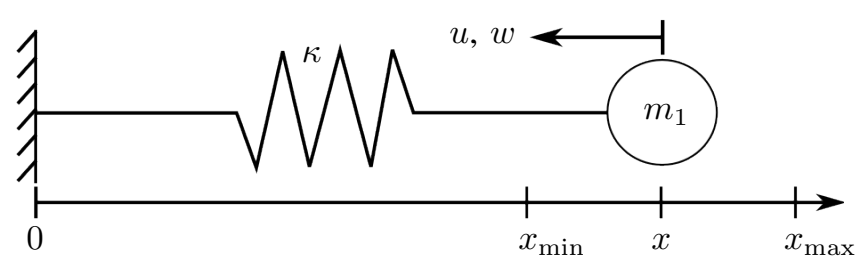

Fig. 1. Dog walking schematic. is in the field. Any position $x_{\min } \leq x \leq x_{\max }$ is acceptable. Furthermore any speed $|\dot{x}| \leq 1 \mathrm{~m} \mathrm{~s}^{-1}$ is allowed. The force the man exerts on himself is denoted by $u$, and must satisfy $|u| \leq 0.5 m_{1} g$, with $g=9.82 \mathrm{~m} \mathrm{~s}^{-2}$, i.e. the man can exert a force of half his body weight in either direction. The desire of the dog to attack the cat is denoted by the linear spring with $\kappa=2 m_{2} g / x_{\min }=32.73 \mathrm{~N} \mathrm{~m}^{-1}$, i.e. when the man is at the edge of the sidewalk the dog is able to exert a steady state force of twice her body weight on the man. In addition to this steady state force the dog is able to exert an unpredictable disturbance force $w$, which is bounded by $0 \leq w \leq m_{2} g$, i.e. positive and less than the body weight of the dog. The continuous-time dynamics are given by

$\left[\begin{array}{l}\dot{x} \\ \ddot{x}\end{array}\right]=\left[\begin{array}{cc}0 & 1 \\ \frac{-\kappa}{m_{1}} & 0\end{array}\right]\left[\begin{array}{l}x \\ \dot{x}\end{array}\right]+\left[\begin{array}{c}0 \\ \frac{-1}{m_{1}}\end{array}\right] u+\left[\begin{array}{c}0 \\ \frac{-1}{m_{1}}\end{array}\right] w$

and are discretized with a step-size of $0.1 \mathrm{~s}$.

The objective is to design a robust MPC controller for the force input $u$ the man applies to himself using $R=1$ and $Q=0.1 I_{2}$. This implies the man is mostly interested in using a low amount of energy, but does make some effort to move towards the cat. The cat remains seated at the origin throughout the entire course of events.

The MCI and MRCI sets for this problem are shown in Figure 2 and 3 by the lighter and darker sets, respectively.

Six control laws were considered. Cost matrices $R=1$, $Q=0.1 I_{2}$ and horizon $N=15$ were employed for each:

ADF: Affine disturbance feedback. This is the standard affine disturbance feedback closed-loop MPC strategy. The feedback policy $(\mathbf{M}, \mathbf{h})$ is optimized. There are $N m+$ $\frac{N(N-1) m p}{2}$ optimization variables.

OLP: Open-loop prediction. This is the standard robust open-loop MPC strategy. The predicted control input trajectory $\mathbf{u}_{i}$ is directly optimized. There are $N m$ optimization variables.

ADF-R: Relaxed affine disturbance feedback. This is the non-standard affine disturbance feedback closed-loop MPC strategy proposed in this paper. It is equivalent to setting blocking matrix $\Lambda=I$. The feedback policy $(\mathbf{M}, \mathbf{h})$ is optimized. There are $N m+\frac{N(N-1) m p}{2}$ optimization variables.

ADF-D: Diagonally blocked affine disturbance feedback. This is the non-standard affine disturbance feedback closedloop MPC strategy proposed in this paper. It employes the diagonal blocking strategy mentioned in Remark 4. The feedback policy $(\tilde{\mathbf{M}}, \mathbf{h})$ is optimized. There are $N m+(N-1) m p$ optimization variables.

ADF-1: One degree of freedom blocked affine disturbance feedback. This is the non-standard affine disturbance feedback closed-loop MPC strategy proposed in this paper. It employes a blocking matrix $\Lambda=[1, \ldots, 1]^{\mathrm{T}}$, i.e. $\nu=1$. This implies that each decision variable of $\mathbf{M}$ is forced to be the same. The feedback policy $(\tilde{\mathbf{M}}, \mathbf{h})$ is optimized. There are $N m+1$ optimization variables.

ADF-0: Zero degree of freedom blocked affine disturbance 


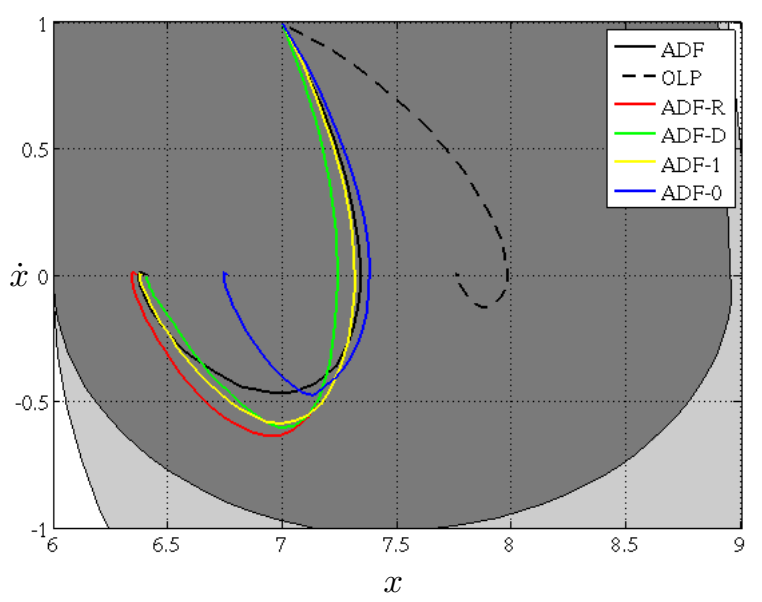

Fig. 2. Nominal trajectories with proposed controllers.

feedback. This is the non-standard affine disturbance feedback closed-loop MPC strategy proposed in this paper. It employes a blocking matrix $\Lambda=0$. This implies $\mathbf{M}=0$, i.e. open-loop prediction. Only offset vector $\mathbf{h}$ is optimized. There are $\mathrm{Nm}$ optimization variables.

Note that each of these strategies has the same number of prediction state and input constraints. Further note that the structure of $\{\mathrm{OLP}, \mathrm{ADF}\}$ and ADF-R, ADF-D, ADF-1, ADF- 0$\}$ are different. In the former the terminal state, in the latter the state of the first prediction step, is constrained to the MRCI set. State constraint relaxations are applied for all strategies in the latter.

Plotted in Figure 2 are six state trajectories, one for each of the six control strategies, from initial state $x_{0}=(7,1)$. These simulations were performed without noise. This is to check the nominal performance of the controllers. Denote by $\bar{x}$ and $\bar{u}$ the steady-state state and control input, respectively, reached after 500 steps. Denote by $T$ the average computation time per iteration, averaged over the 500 simulation steps. Further denote by $V / V_{\mathrm{ADF}}$ the ratio of steady-state cost of the particular controller w.r.t. the steady-state cost of the ADF controller. The following results are obtained:

\begin{tabular}{lcccc} 
& $\bar{x}$ & $\bar{u}$ & $T$ & $V / V_{\text {ADF }}$ \\
\hline ADF: & 6.40 & -209.6 & 0.710 & 1.000 \\
OLP: & 7.76 & -254.1 & 0.571 & 1.470 \\
ADF-R: & 6.36 & -208.2 & 0.611 & 0.986 \\
ADF-D: & 6.41 & -209.7 & 0.585 & 1.001 \\
ADF-1: & 6.38 & -209.0 & 0.578 & 0.994 \\
ADF-0: & 6.75 & -221.1 & 0.572 & 1.113
\end{tabular}

In Figure 3 all settings are kept the same except for the fact that a predefined disturbance sequence is applied. Here no steady state is reached since there is a persistent disturbance. All trajectories stay within the MRCI set.

\section{CONCLUSION}

In this paper it was shown that by employing blocking the computational complexity of affine disturbance feedback can be reduced, especially for long prediction horizons, while retaining good performance and guaranteeing strong feasibility. Strong feasibility is guaranteed by enforcing the first

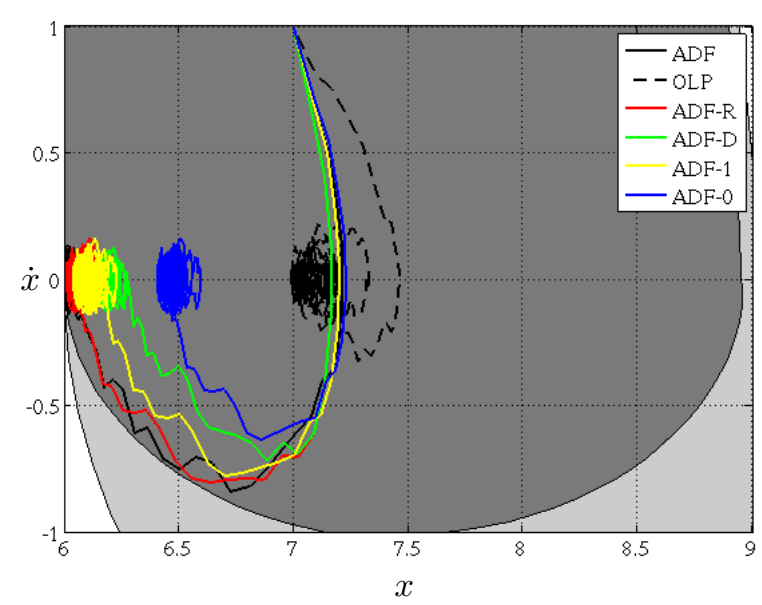

Fig. 3. Disturbed trajectories with proposed controllers.

predicted state to lie within the robust controlled invariant feasible set. For this an existing method was extended to the robust case and adapted for using with affine disturbance feedback. Furthermore, it was shown that by imposing a structure on the feedback matrix the number of decision variables is reduced and so is subsequently the computational effort. Even with the limited degree of freedom, the performance achieved by blocked affine disturbance feedback is still remarkably good and close to the unblocked case.

\section{REFERENCES}

[1] A. Ben-Tal, A. Goryashko, E. Guslitzer, and A. Nemirovski. Adjustable robust solutions of uncertain linear programs. Math. Program., 99(2):351-376, 2004.

[2] F. Blanchini. Ultimate boundedness control for uncertain discrete time systems via set-induced lyapunov functions. IEEE Trans. Automatic Control, 39(2):428-433, 1994.

[3] F. Blanchini. Set invariance in control. Automatica, 35(11):1747-1767, 1999.

[4] R. Cagienard, P. Grieder, E.C. Kerrigan, and M. Morari. Move blocking strategies in receding horizon control. Journal of Process Control, 17:563-570, 2007.

[5] C.E.T. Dórea and J.C. Hennet. (a,b)-invariant polyhedral sets of linear discrete-time systems. J. Optim. Theory Appl., 103(3):521-542, 1999.

[6] R. Gondhalekar and J. Imura. Least restrictive move-blocking model predictive control. In Proc. IFAC World Congress, pages 11190-11195, 2008.

[7] R. Gondhalekar, J. Imura, and K. \& Kashima. Rigorous determination of maximum controlled invariant feasible sets. In Proc. 10th EUCA European Control Conference, 2009.

[8] P.J. Goulart, E.C. Kerrigan, and J.M. Maciejowski. Optimization over state feedback policies for robust control with constraints. Automatica, 42(4):523-533, April 2006.

[9] J. Loefberg. Approximations of closed-loop minimax mpc. In Proc. 42nd IEEE Conference on Decision and Control, pages 1438-1442, 2003.

[10] D. Q. Mayne, J. B. Rawlings, C. V. Rao, and P. O. M. Scokaert. Constrained model predictive control: Stability and optimality. Automatica, 36(6):789-814, June 2000.

[11] F. Oldewurtel, C.N. Jones, and M. Morari. A tractable approximation of chance constrained stochastic mpc based on affine disturbance feedback. In Proc. 47th IEEE Conference on Decision and Control, pages 4731-4736, 2008. 